\title{
Homogeneous fluid turbulence before the final period of decay for four-point correlation in a rotating system for first-order reactant
}

\author{
M. Monuar Hossain ${ }^{1}$, M. Abu Bkar Pk ${ }^{2, ~ *}$, M. S. Alam Sarker ${ }^{2}$ \\ ${ }^{1}$ Department of Civil \& Environmental Engineering, Uttra University, Dhaka-1230, Bangladesh \\ ${ }^{2}$ Department of Applied Mathematics, University of Rajshahi, Rajshahi-6205, Bangladesh
}

Email address:

milon4215@gmail.com (M. M. Hossain), abubakarpk_ru@yahoo.com (M. A. Bkar Pk), sasmathbd@yahoo.com (M. S. A. Sarker)

\section{To cite this article:}

M. Monuar Hossain, M. Abu Bkar Pk, M. S. Alam Sarker. Homogeneous Fluid Turbulence before the Final Period of Decay for Four-Point Correlation in a Rotating System for First-Order Reactant. American Journal of Theoretical and Applied Statistics. Vol. 3, No. 4, 2014, pp. 81-89. doi: 10.11648/j.ajtas.20140304.11

\begin{abstract}
Following Deissler's approach, the decay in homogeneous turbulence at times preceding to the ultimate phase for the concentration fluctuation of a dilute contaminant undergoing a first-order chemical reaction in a rotating system for the case of four-point correlation is studied. Two-, three-, and four-point correlation equations have been obtained and the correlation equations are converted to spectral form by their Fourier-transform, the terms containing quintuple correlations are neglected in comparison to the third and fourth order correlation terms. Finally, integrating the energy spectrum over all wave numbers, the energy decay law of homogeneous turbulent flow for the concentration fluctuations ahead of the ultimate phase in a rotating system for four-point correlation has been obtained and it is shown by graphically in the text.
\end{abstract}

Keywords: Chemical Reactant, Deissler's Method, Fourier-Transformation, Navier-Stokes Equation, Turbulent Flow, Correlation Function

\section{Introduction}

Deissler $(1958,1960)$ developed a theory 'on the decay of homogeneous turbulence before the final period. Using Deissler's theory, Kumar and patel (1974) studied the firstorder reactant in homogeneous turbulence before the final period of decay for the case of multi-point and single- time correlation. Kumar and Patel (1975) extended their problem for the case of multi-point and multi- time concentration correlation. Loeffler and Deissler (1961) studied the decay of temperature fluctuations in homogeneous turbulence before the final period. Batchelor (1953) studied the theory of homogeneous turbulence. Bkar Pk et al. (2012) studied the decay of energy of MHD turbulence for four point correlation. Aziz et al. (2010) obtained the first order reactant in MHD turbulence before the final period of decay for the case of multi-point and multi-time in a rotating system in presence of dust particle. Bkar Pk et al (2013(a)) furthermore considered the first order reactant in homogeneous turbulence prior to the ultimate phase of decay for four point correlation in presence of dust particle.
Chandrasekhar (1951) obtained the invariant theory of isotropic turbulence in magneto-hydrodynamics. Sarker and Kishore (1991) studied the decay of MHD turbulence before the final period. Sarker and Islam (2001) obtained the decay of dusty fluid MHD turbulence before the final period in a rotating system. Bkar Pk et al (2013(b)) further calculated the decay of MHD turbulence before the final period for four-point correlation in a rotating system. Mondal (2006) discussed the energy decay law of dusty fluid turbulent flow in a rotating system. Shamima Sultan (2008) studied the energy decay law of dusty fluid turbulent flow in a rotating system.

In this paper, following Deissler's theory we have studied the first-order reactant in homogeneous turbulence prior to the ultimate phase of decay for four-point correlation in a rotating system under the restrictions that the turbulence and the concentration fields are homogeneous; the reaction rate and the diffusivity are constant. This study shows that the chemical reaction causes the concentration fluctuation to decay more rapidly than they would for non pure mixing. 


\section{Basic Equation}

The differential equation governing the concentration of a dilute contaminant undergoing a first-order chemical reaction in homogeneous fluid turbulence in a rotating system could be written as

$$
\frac{\partial u_{i}}{\partial t}+u_{k} \frac{\partial u_{i}}{\partial x_{k}}=D \frac{\partial^{2} u_{i}}{\partial x_{k} \partial x_{k}}-R u_{i}-2 \varepsilon_{m k i} \Omega_{m} u_{i}
$$

where $u_{i}(\hat{x})$ is a random function of position and time at a point $\mathrm{p}, u_{k}(\hat{x}, t)$ is the turbulent velocity, $R$ is the constant reaction rate, $D$ is the diffusivity, $t$ is the time, $\mathcal{E}_{m k i}$ is the alternating tensor, $\Omega_{m}$ is the angular velocity components, $u_{i}$ is the turbulent velocity component, $x_{k}$ is the space coordinate, and repeated subscript in a term indicates a summation of terms, with the subscripts successively taking on the values $1,2,3$.

\section{Two-Point Correlation and Spectral Equations}

Under the restrictions that the turbulence and the concentration fields are homogeneous, the chemical reaction and the local mass transfer have no effect on the velocity field and the reaction rate and the diffusivity are constant. The differential equation of a dilute contaminant undergoing a first-order chemical reaction in homogeneous system could be written as

$$
\frac{\partial \Gamma}{\partial t}+u_{k} \frac{\partial \Gamma}{\partial x_{k}}=D \frac{\partial^{2} \Gamma}{\partial x_{k} \partial x_{k}}-R \Gamma
$$

Subtracting the mean of (2) from Equ. (2), we obtain

$$
\begin{aligned}
& \frac{\partial X}{\partial t}+u_{k} \frac{\partial X}{\partial x_{k}}=D \frac{\partial^{2} X}{\partial x_{k} \partial x_{k}}-R X \\
& \frac{\partial\left\langle u_{i} X^{\prime} X^{\prime \prime}\right\rangle}{\partial t}+\frac{\partial\left\langle u_{k} u_{k}^{\prime} X^{\prime} X^{\prime \prime}\right\rangle}{\partial x_{k}}+\frac{\partial\left\langle u_{i} u_{k}^{\prime} X^{\prime} X^{\prime \prime}\right\rangle}{\partial x_{k}^{\prime}}+\frac{\partial\left\langle u_{i} u_{k}^{\prime \prime} X^{\prime} X^{\prime \prime}\right\rangle}{\partial x_{k}^{\prime \prime}} \\
& =-\frac{1}{\rho} \frac{\partial\left\langle p X^{\prime} X^{\prime \prime}\right\rangle}{\partial x_{i}}+v \frac{\partial^{2}\left\langle u_{i} X^{\prime} X^{\prime \prime}\right\rangle}{\partial x_{k} \partial x_{k}} \\
& +D\left(\frac{\partial^{2}}{\partial x_{k} \partial x_{k}}+\frac{\partial^{2}}{\partial x_{k}^{\prime} \partial x_{k}^{\prime}}\right)\left\langle u_{i} X^{\prime} X^{\prime \prime}\right\rangle-2 R\left\langle u_{i} X^{\prime} X^{\prime \prime}\right\rangle-2 \varepsilon_{m k i} \Omega_{m}\left\langle u_{i} X^{\prime} X^{\prime \prime}\right\rangle
\end{aligned}
$$

where $X(\hat{x}, t)$ is the fluctuation of concentration about the mean at a point $p(\hat{x})$ and time $t$. The two-point correlation for the fluctuating concentration can be written, as

$$
\frac{\partial\left\langle X X^{\prime}\right\rangle}{\partial t}+u_{k} \frac{\partial\left\langle X X^{\prime}\right\rangle}{\partial x_{k}}=D \frac{\partial^{2}\left\langle X X^{\prime}\right\rangle}{\partial x_{k} \partial x_{k}}-R\left\langle X X^{\prime}\right\rangle
$$

where the fluctuating concentration at the point and the point $p^{\prime}$ is at a distance $\mathrm{r}$ from the point $\mathrm{p}$. The symbol \langle\rangle is the ensemble average. Putting the Fourier transforms

$$
\begin{aligned}
\left\langle\mathrm{XX}^{\prime}(\mathrm{r})\right\rangle & =\int_{-\infty}^{\infty} \theta(\hat{K}, t) \exp [i((\hat{K}, \hat{r})] d \hat{K}, \\
\left\langle\mathrm{u}_{\mathrm{k}} \mathrm{XX}^{\prime}(\mathrm{r})\right\rangle & =\int_{-\infty}^{\infty} \phi_{k}(\hat{K}, t) \exp [i((\hat{K}, \hat{r})] d \hat{K}, \\
\left\langle\mathrm{u}_{\mathrm{k}}^{\prime} \mathrm{XX}^{\prime}(\mathrm{r})\right\rangle & =\int_{-\infty}^{\infty} \phi_{k}(\hat{K}, t) \exp [i((\hat{K}, \hat{r})] d \hat{K},
\end{aligned}
$$

into equation (4), we obtains

$$
\frac{\partial \theta}{\partial t}+\left(D k^{2}+2 R\right) \theta=i k_{k}\left[\varphi_{k}(\hat{K}, t)-\varphi_{k}(-\hat{K}, t)\right]
$$

\section{Three-Point Correlation and Spectral Equation}

Before By taking the Navier-Stokes equations for a first-order chemical reaction in homogeneous fluid turbulence in a rotating system at $p$ is

$$
\frac{\partial u_{i}}{\partial t}+u_{k} \frac{\partial u_{i}}{\partial x_{k}}=D \frac{\partial^{2} u_{i}}{\partial x_{k} \partial x_{k}}-R u_{i}-2 \varepsilon_{m k i} \Omega_{m} u_{i}
$$

and the fluctuation equations at $p^{\prime} \& p^{\prime \prime}$ one can find the
Using the transformations

$$
\frac{\partial}{\partial x_{k}}=-\left(\frac{\partial}{\partial r_{k}}+\frac{\partial}{\partial r_{k}^{\prime}}\right), \frac{\partial}{\partial x_{k}^{\prime}}=\frac{\partial}{\partial x_{k}}, \frac{\partial}{\partial x_{k}^{\prime \prime}}=\frac{\partial}{\partial r_{k}^{\prime}},
$$

In order to convert Eq. (9) to spectral form, using six-dimensional Fourier transforms (Kumar and Patel, 1974) and with the fact that $\left\langle u_{i} u_{k}^{\prime \prime} X^{\prime} X^{\prime \prime}\right\rangle=\left\langle u_{i} u_{k}^{\prime} X^{\prime} X^{\prime \prime}\right\rangle$, we can write equation (9) in the form

$$
\begin{aligned}
& \frac{\partial \beta_{j}}{\partial t}+\left[v\left(\hat{K}+\hat{K}^{\prime}\right)^{2}+D\left(\hat{K}+\hat{K}^{\prime}\right)+2 R+2 \varepsilon_{m k i} \Omega_{m}\right] \beta_{j} \\
& =-i\left(k_{k}+k_{k}^{\prime}\right) \psi_{j k}+i\left(k_{k}+k_{k}^{\prime}\right) \psi_{j k^{\prime}}+\frac{1}{\rho} i\left(k_{j}+k_{j}^{\prime}\right) \gamma
\end{aligned}
$$

where $\mu_{i}\left(\hat{K}, \hat{K}^{\prime}, t\right)=L \beta_{j}\left(\hat{K}, \hat{K}^{\prime}, t\right)$.If the momentum equation (3) at $\mathrm{p}$ is multiplied by $X^{\prime} X^{\prime \prime}$ and divergence of the time averages is taken, the resulting equation will be 


$$
\frac{\partial^{2}\left\langle u_{i} u_{k} X^{\prime} X^{\prime \prime}\right\rangle}{\partial x_{i} \partial x_{k}}=-\frac{1}{\rho} \frac{\partial^{2}\left\langle p X^{\prime} X^{\prime \prime}\right\rangle}{\partial x_{i} \partial x_{i}}
$$

In Fourier space it can be written as

$$
-\left(k_{k}+k_{k}^{\prime}\right) \psi_{i k^{\prime}}=\frac{1}{\rho}\left(k_{i}+k_{i}^{\prime}\right) \gamma .
$$

Substituting this into equation (10), we obtain

$$
\frac{\partial \beta_{j}}{\partial t}+\left[v\left(\hat{K}+\hat{K}^{\prime}\right)^{2}+D\left(\hat{K}+\hat{K}^{\prime}\right)+2 R+2 \varepsilon_{m k i} Q_{m}\right] \beta_{j}=-i\left(k_{k}+k_{k}^{\prime}\right) \psi_{j k}^{\prime}
$$

\section{Four-Point Correlation and Spectral Equation}

and the concentration equations at $p^{\prime \prime}, p^{\prime \prime \prime}$, and following the same procedure as before, one can get the four-point correlations as

Again by taking the Navier-Stokes equations at $\mathrm{p}$ and $p^{\prime}$

$$
\begin{aligned}
& \frac{\partial\left\langle u_{i} u_{j}^{\prime} X^{\prime \prime} X^{\prime \prime \prime}\right\rangle}{\partial t}+\frac{\partial\left\langle u_{i} u_{k} u_{j}^{\prime} X^{\prime \prime} X^{\prime \prime \prime}\right\rangle}{\partial x_{k}}+\frac{\partial\left\langle u_{j}^{\prime} u_{k}^{\prime \prime} u_{i} X^{\prime \prime} X^{\prime \prime \prime}\right\rangle}{\partial x_{k}^{\prime}}+\frac{\partial\left\langle u_{i} u_{j}^{\prime} u_{k}^{\prime \prime} X^{\prime \prime} X^{\prime \prime \prime}\right\rangle}{\partial x_{k}^{\prime \prime}}+\frac{\partial\left\langle u_{i} u_{j}^{\prime} u_{k}^{\prime \prime \prime} X^{\prime \prime} X^{\prime \prime \prime}\right\rangle}{\partial x_{k}^{\prime \prime}} \\
= & -\frac{1}{\rho}\left[\frac{\partial\left\langle p u_{j}^{\prime} X^{\prime \prime} X^{\prime \prime \prime}\right\rangle}{\partial x_{i}}+\frac{\partial\left\langle p^{\prime} u_{i} X^{\prime \prime} X^{\prime \prime \prime}\right\rangle}{\partial x_{j}^{\prime}}\right]+v\left(\frac{\partial^{2}}{\partial x_{k} \partial x_{k}}+\frac{\partial^{2}}{\partial x_{k}^{\prime} x_{k}^{\prime}}\right)\left\langle u_{i} u_{j}^{\prime} X^{\prime \prime} X^{\prime \prime \prime}\right\rangle+D\left(\frac{\partial^{2}}{\partial x_{k}^{\prime \prime} \partial x_{k}^{\prime \prime}}+\frac{\partial^{2}}{\partial x_{k}^{\prime \prime \prime} \partial x_{k}^{\prime \prime \prime}}\right)\left\langle u_{i} u_{j}^{\prime} X^{\prime \prime} X^{\prime \prime \prime}\right\rangle \\
& -2 R\left\langle u_{i} u_{j}^{\prime} X^{\prime \prime} X^{\prime \prime \prime}\right\rangle-2 \varepsilon_{m k i} \Omega_{m}\left\langle u_{i} u_{j}^{\prime} X^{\prime \prime} X^{\prime \prime \prime}\right\rangle
\end{aligned}
$$

Using the transformations,

$$
\frac{\partial}{\partial x_{k}}=-\left(\frac{\partial}{\partial r_{k}}+\frac{\partial}{\partial r_{k}^{\prime}}++\frac{\partial}{\partial r_{k}^{\prime \prime}}\right), \frac{\partial}{\partial x_{k}^{\prime}}=\frac{\partial}{\partial x_{k}}, \frac{\partial}{\partial x_{k}^{\prime \prime}}=\frac{\partial}{\partial r_{k}^{\prime}}, \frac{\partial}{\partial x_{k}^{\prime \prime \prime}}=\frac{\partial}{\partial r_{k}^{\prime \prime}}
$$

In order to convert Eq. (14) to spectral form, using nine-dimensional Fourier transforms (Kumar and Patel, 1974) and with the fact that

$$
\left\langle u_{k}^{\prime \prime \prime} u_{i} u_{j}^{\prime} X^{\prime \prime \prime} X^{\prime \prime}\left(\hat{r}, \hat{r}^{\prime}, \hat{r}^{\prime \prime}\right)\right\rangle=\left\langle u_{k}^{\prime \prime} u_{i} u_{j}^{\prime} X^{\prime \prime} X^{\prime \prime \prime}\left(\hat{r}, \hat{r}^{\prime}, \hat{r}^{\prime \prime}\right)\right\rangle
$$

Equation (14) can be written as

$$
\begin{aligned}
& \frac{\partial g_{i j}}{\partial t}+\left[v\left(\hat{K}+\hat{K}^{\prime}+\hat{K}^{\prime \prime}\right)^{2}+v \hat{K}^{2}+D\left(\hat{K}^{\prime}+\hat{K}^{\prime \prime}\right)^{2}+2 R+2 \varepsilon_{m k i} \Omega_{m}\right] g_{i j} \\
& =-i\left(k_{k}+k_{k}^{\prime}+k_{k}^{\prime \prime}\right)\left[h_{i j k}\left(\hat{K}, \hat{K}^{\prime}, \hat{K}^{\prime \prime}\right)-i k_{k}\left[h_{i j k}\left(-\hat{K}-\hat{K}^{\prime}-\hat{K}^{\prime \prime}, \hat{K}^{\prime}, \hat{K}^{\prime \prime}\right)\right]\right. \\
& -i\left(k_{k}^{\prime}+k_{k}^{\prime \prime}\right) h_{i j k}\left(\hat{K}, \hat{K}^{\prime}, \hat{K}^{\prime \prime}\right)-\frac{1}{\rho}\left[-\left(k_{i}+k_{i}^{\prime}+\overline{k_{i}^{\prime \prime}}\right) \Omega_{j}\left(\hat{K}, \hat{K}^{\prime}, \hat{K}^{\prime \prime}\right)+k_{j}^{\prime} \Omega_{i}\left(-\hat{K}-\hat{K}^{\prime}-\hat{K}^{\prime \prime}, \hat{K}^{\prime}, \hat{K}^{\prime \prime}\right)\right] .
\end{aligned}
$$

where $\mu_{i j}\left(\hat{K}, \hat{K}^{\prime}, \hat{K}^{\prime \prime}, t\right)=M g_{i j}\left(\hat{K}, \hat{K}^{\prime}, \hat{K}^{\prime \prime}, t\right)$. Following the same procedure as was used in obtaining (12), we get

$$
\Omega_{j}\left(\hat{K}, \hat{K}^{\prime}, \hat{K}^{\prime \prime}\right)=-\rho \frac{\left(k_{k}+k_{k}^{\prime}+k_{k}^{\prime \prime}\right)}{\left(k_{i}+k_{i}^{\prime}+k_{i}^{\prime \prime}\right)} h_{i j k}\left(\hat{K}, \hat{K}^{\prime}, \hat{K}^{\prime \prime}\right) .
$$

Equation (15) and (16) are the spectral equations corresponding to the four-point correlations. To get a better picture of the of the first-order reactant of homogeneous turbulence decay in a rotating system from its initial period to its final period, four-point correlations are to be considered. The same ideology could be applied to the concentration phenomenon. Here, we neglect the quintuple correlations since the decay faster than the lower-order correlations. As pointed out by Deissler $(1958,1960)$ when the quintuple correlations are neglected, the corresponding pressure-force terms which are related to them are also neglected. Under these assumptions, equations, (15) and (16) give the solution as

$$
\begin{aligned}
g_{i j}\left(\hat{K}, \hat{K}^{\prime}, \hat{K}^{\prime \prime}, t\right)= & {\left[g _ { i j } ( \hat { K } , \hat { K } ^ { \prime } , \hat { K } ^ { \prime \prime } , t _ { 0 } ) \operatorname { e x p } \left\{-D\left[2 N_{s} k^{2}+\left(1+N_{s}\right)\left(k^{2}+k^{\prime \prime 2}\right)\right.\right.\right.} \\
& \left.\left.+2 N_{s}\left(k_{k} k_{k}^{\prime}+k_{k}^{\prime} k_{k}^{\prime \prime}+k_{k} k_{k}^{\prime \prime}\right)\right]-2 R+2 \varepsilon_{m k i} \Omega_{m}\right\}\left(t-t_{0}\right)
\end{aligned}
$$


$N_{s}=v / D$.A relation between $g_{i j}\left(\hat{K}, \hat{K}^{\prime}, \hat{K}^{\prime \prime}, t_{0}\right)$ and $\varphi_{i j}^{\prime}\left(\hat{K}, \hat{K}^{\prime}\right)$ can be found by taking $\hat{r}^{\prime \prime}=0$ in the expression for $\left\langle u_{i} u_{j}^{\prime} X^{\prime \prime} X^{\prime \prime \prime}\left(\hat{r}, \hat{r}^{\prime}, \hat{r}^{\prime \prime}\right)\right\rangle$ and comparing it with the expression for $\left\langle u_{i} u_{k}^{\prime} X^{\prime} X^{\prime \prime}\left(\hat{r}, \hat{r}^{\prime}\right)\right\rangle$.

$$
\varphi_{i k}^{\prime}\left(\hat{K}, \hat{K}^{\prime}\right)=\int_{-\infty}^{\infty} g_{i j}\left(-\hat{K}-\hat{K}^{\prime}, \hat{K}^{\prime}, \hat{K}^{\prime \prime}, t\right) d \hat{K}^{\prime \prime} .
$$

Substituting this in to equation (13), we obtains

$$
\begin{aligned}
& \frac{\partial \beta_{j}}{\partial t}+D\left[\left(1+N_{s}\right)\left(K^{2}+K^{\prime 2}\right)+2 N_{s} \hat{K} \cdot \hat{K}^{\prime}+\frac{2 R}{D}+\frac{2 \varepsilon_{m k i} \Omega_{m}}{D}\right] \beta_{j} \\
& =-2 i\left(k_{k}+k_{k}^{\prime}\right)\left[g_{i j}\left(-\hat{K}-\hat{K}^{\prime}, \hat{K}^{\prime}, \hat{K}^{\prime \prime}, t_{0}\right)\right]_{0} \\
& \times \frac{a \pi^{3 / 2}}{D^{3 / 2}\left(1+N_{s}\right)^{3 / 2}\left(t-t_{0}\right)^{3 / 2}} \times \exp \left\{-D\left[\frac{N_{s}\left(2+N_{s}\right) k^{2}}{\left(1+N_{s}\right)}+2 N_{s} k k^{\prime}+\left(1+N_{s}\right) k^{\prime 2}+\frac{2 R}{D}+\frac{2 \varepsilon_{m k i} \Omega_{m}}{D}\right]\right\}\left(t-t_{0}\right) .
\end{aligned}
$$

Now, the solution of equation (19) is

$$
\begin{gathered}
\left.\beta_{j}\left(\hat{K}, \hat{K}^{\prime}, t_{0}\right)=\mid \beta_{j}\left(\hat{K}, \hat{K}^{\prime}, t_{0}\right)\right]_{0} . \\
\times \exp \left\{-D\left[\left(1+N_{s}\right)\left(K^{2}+K^{\prime 2}\right)+2 N_{s} \hat{K} . \hat{K}^{\prime}+\left(\frac{2 R}{D}+\frac{2 \varepsilon_{m k i} \Omega_{m}}{D}\right)\right]\left(t-t_{0}\right)\right\} \\
-\frac{2 i\left(k_{k}+k_{k}^{\prime}\right) b_{i} \pi^{3 / 2}}{D^{\frac{3}{2}}\left(1+N_{s}\right)^{3 / 2}} \times\left[\frac{-2}{\left(t-t_{0}\right)^{3 / 2}}+\frac{4 k D^{1 / 2} F(\omega)}{\left(1+N_{s}\right)^{1 / 2}}\right] \\
\times \exp \left\{-D\left[\frac{N_{s}\left(2+N_{s}\right) k^{2}}{\left(1+N_{s}\right)}+2 N_{s} k k^{\prime}+\left(1+N_{s}\right) k^{\prime 2}\right.\right. \\
\left.\left.+\left(\frac{2 \varepsilon_{m k i} \Omega_{m}}{D}\right)\right]\right\}\left(t-t_{0}\right) \times \exp \left[-2 R\left(t-t_{0}\right)\right]
\end{gathered}
$$

where

$$
\begin{gathered}
F(\omega)=\exp \left(-\omega^{2}\right) \int_{0}^{\infty} \exp \left(n^{2}\right) d n, \omega=k\left[\frac{\left(t-t_{0}\right) D}{\left.91+N_{s}\right)}\right]^{1 / 2}, \text { and } \\
{\left[g_{i j}\left(-\hat{K}-\hat{K}^{\prime}, \hat{K}^{\prime}, \hat{K}^{\prime \prime}, t_{0}\right)\right]_{0}=b_{i}}
\end{gathered}
$$

Now, by taking $\hat{r}^{\prime}=0$ in the expression for $\left\langle u^{\prime} X^{\prime} X^{\prime \prime \prime}\left(\hat{r}, \hat{r}^{\prime}\right\rangle\right.$ is performed, we obtain and comparing the result with the expression $\left\langle X u_{k}^{\prime} X^{\prime}(\hat{r})\right\rangle$,

$$
\varphi_{k}(\hat{K})=\int_{\infty}^{\infty} \beta_{k}\left(\hat{K} \cdot \hat{K}^{\prime}\right) d k^{\prime}
$$

Substituting equation (21) into equation (8), we obtain

$$
\frac{\partial G}{\partial t}+\left(2 D k^{2}+2 R\right) G=W
$$

where $G=2 \pi k^{2} \theta$ and

$$
\begin{gathered}
W=2 \pi i k^{2} \exp \left[-2 R\left(t-t_{0}\right)\right] \\
\times \int_{-\infty}^{\infty} k_{k}\left[\beta_{k}\left(\hat{K}, \hat{K}^{\prime}\right)-\beta_{k}\left(-\hat{K},-\hat{K}^{\prime}\right)\right]_{0} \\
\times \exp \left\{-D\left[\left(1+N_{s}\right)\left(k^{2}+k^{\prime 2}\right)\right.\right. \\
\left.\left.+2 N_{s} \hat{K} \cdot \hat{K}^{\prime}+\left(\frac{2 \varepsilon_{m k i} \Omega_{m}}{D}\right)\right]\left(t-t_{0}\right)\right\} \\
+\frac{2 i \pi^{5 / 2}}{D^{3 / 2}\left(1+N_{s}\right)^{3 / 2}} \int_{-\infty}^{\infty}\left[b_{i}\left(\hat{K}, \hat{K}^{\prime}\right)-b_{i}\left(-\hat{K},-\hat{K}^{\prime}\right)\right] \\
\times\left[\frac{-2}{\left(t-t_{0}\right)^{1 / 2}}+\frac{4 k F(w) D^{1 / 2}}{\left(1+N_{s}\right)^{1 / 2}}\right] \\
\times \exp \left\{-D\left[\frac{N_{s}\left(2+N_{s}\right) k^{2}}{\left(1+N_{s}\right)}+2 N_{s} k k^{\prime}+\right.\right. \\
\left.\left.\left(1+N_{s}\right) k^{\prime 2}+\left(\frac{2 \varepsilon_{m k i} \Omega_{m}}{D}\right)\right]\left(t-t_{0}\right) d k^{\prime}\right\} .
\end{gathered}
$$

In analogy with the turbulent energy spectrum function, the quantity $G$, in equation (23) can be called concentration energy spectrum function, and $W$, the energy transfer function, is responsible for the transfer of concentration from one wave number to another. In order to find solution completely and following Deissler's $(1958,1960)$ we assume that

$$
\begin{aligned}
& \left.(2 \pi)^{2} i k_{k} \mid \beta_{0}\left(\hat{K}, \hat{K}^{\prime}\right)-\beta_{0}\left(-\hat{K},-\hat{K}^{\prime}\right)\right\rfloor_{0} \\
& =-\xi_{0}\left(k^{2} k^{\prime 4}-k^{4} k^{2}\right)
\end{aligned}
$$

and

$$
\begin{aligned}
& \frac{8 \pi^{7 / 2}}{D^{3 / 2}\left(1+N_{s}\right)^{3 / 2}} i\left[b\left(\hat{K}, \hat{K}^{\prime}\right)-b\left(-\hat{K},-\hat{K}^{\prime}\right)\right]_{0} \\
& =-\xi_{1}\left(k^{4} k^{\prime 6}-k^{6} k^{\prime 4}\right),
\end{aligned}
$$


where $\xi_{0}$ and $\xi_{1}$ are arbitrary constants depending on the initial conditions. If these results are used in equation (24) and the integration is performed, we obtain

$$
\begin{aligned}
& W=-\frac{\xi_{0} \pi^{1 / 2} N_{s}}{2\left(t-t_{0}\right)^{3 / 2} D^{3 / 2}\left(1+N_{s}\right)^{5 / 2}} \\
& \times \exp \left\{-D\left[\left(\frac{1+2 N_{s}}{1+N_{s}}\right) k^{2}+\left(\frac{2 \varepsilon_{m k i} \Omega_{m}}{D}\right)\right]\left(t-t_{0}\right)\right\} \\
& \times\left\{\frac{15 k^{4}}{4 D^{2} N^{2}{ }_{s}\left(t-t_{0}\right)^{2}}\left(\frac{N_{s}}{1+N_{s}}\right)+\left[5\left(\frac{N_{s}}{1+N_{s}}\right)^{2}-\frac{3}{2}\right] \frac{k^{6}}{N_{s} D\left(t-t_{0}\right)}\right. \\
& \left.+\left[\left(\frac{N_{s}}{1+N_{s}}\right)^{3}-\left(\frac{N_{s}}{1+N_{s}}\right)\right] k^{8}\right\} \\
& +\frac{\xi_{1} \pi^{1 / 2} N_{s}}{\left(t-t_{0}\right)^{7 / 2} D^{3 / 2}\left(1+N_{s}\right)^{7 / 2}} \\
& \times \exp \left\{-2 D\left[\left(\frac{N_{s}}{1+N_{s}}\right) k^{2}+\left(\frac{\varepsilon_{m k i} \Omega_{m}}{D}\right)\right]\left(t-t_{0}\right)\right\} \\
& \times\left\{\frac{105 k^{6}}{8 D^{3} N^{2}\left(t-t_{0}\right)^{3}}\left(\frac{N_{s}}{1+N_{s}}\right)+\frac{15 k^{8}}{4 D^{3} N_{s}\left(t-t_{0}\right)^{2}}\right. \\
& \times\left[7\left(\frac{N_{s}}{1+N_{s}}\right)^{2}-1\right]+\left(\frac{N_{s} k^{10}}{D\left(1+N_{s}\right)\left(t-t_{0}\right)}\right) \\
& \left.\times\left[\frac{21}{2}\left(\frac{N_{s}}{1+N_{s}}\right)^{2}-5\right]+\frac{N^{2} k^{12}}{\left(1+N_{s}\right)^{2}\left(t-t_{0}\right)}\left[\left(\frac{N_{s}}{1+N_{s}}\right)^{2}-1\right]\right\} \\
& -\frac{2 \xi_{1} \pi^{1 / 2} N_{s}}{\left(t-t_{0}\right)^{3 / 2} D\left(1+N_{s}\right)^{4}} \\
& \times \exp \left\{-D\left[\left(\frac{1+2 N_{s}}{1+N_{s}}\right)+\left(\frac{2 \varepsilon_{m k i} \Omega_{m}}{D}\right)\right]\left(t-t_{0}\right)\right\} \\
& \times\left\{\frac{105 k^{7}}{8 D^{3} N^{2}{ }_{s}\left(t-t_{0}\right)^{3}}\left(\frac{N_{s}}{1+N_{s}}\right)+\frac{15 k^{9}}{4 D^{3} N_{s}\left(t-t_{0}\right)^{2}}\right. \\
& \times\left[7\left(\frac{N_{s}}{1+N_{s}}\right)^{2}-1\right]+\left(\frac{N_{s} k^{11}}{D\left(1+N_{s}\right)\left(t-t_{0}\right)}\right) \\
& \times\left[\frac{21}{2}\left(\frac{N_{s}}{1+N_{s}}\right)^{2}-5\right]+\frac{N^{3} k^{13}}{\left(1+N_{s}\right)^{2}}\left[\left(\frac{N_{s}}{1+N_{s}}\right)^{2}-1\right] \\
& \left.\times \int_{0}^{\omega} \exp \left(n^{2}\right) d n\right\} \exp \left[-2 R\left(t-t_{0}\right)\right]
\end{aligned}
$$

It is very interesting that,

$$
\int_{-\infty}^{\infty} W d k=0
$$

This indicates that the expression for $W$ satisfies the condition of continuity and homogeneity. Physically it was to be expected, since $W$ is the measure of transfer of energy and the total energy transformed to all wave numbers is to be zero, which is what equation (26) gives. The linear equation (23) can be solved to give

$$
\begin{aligned}
& G=J(k) \exp \left[-\left(2 D k^{2}+2 R\right)\left(t-t_{0}\right)\right] \\
& +\exp \left[-\left(2 D k^{2}+2 R\right)\left(t-t_{0}\right)\right] \int W \\
& \times \exp \left[-\left(2 D k^{2}+2 R\right)\left(t-t_{0}\right)\right] d t
\end{aligned}
$$

where $J(k)=\frac{N_{0} k^{2}}{\pi}$ is a constant of integration and can be obtained as by (Corrsin,1951). Thus, by integrating the right-hand side of equation, we obtain

$$
\begin{aligned}
& G=\exp \left[-2 R\left(t-t_{0}\right)\left\{\frac{N_{0}\left(1+N_{s}\right) \varepsilon^{2}}{D \pi\left(t-t_{0}\right)}\right.\right. \\
& \times \exp \left[-2\left(1+N_{s}\right) \eta^{2}+\left(\frac{2 \varepsilon_{m k i} \Omega_{m}}{D}\right)\right] \\
& +\frac{\xi_{0} \pi^{1 / 2} N_{s}}{2\left(t-t_{0}\right)^{9 / 2} D^{11 / 2} 2\left(1+N_{s}\right)^{3 / 2}} \\
& \times \exp \left[-\left(1+2 N_{s}\right) \varepsilon^{2}+\left(\frac{2 \varepsilon_{m k i} \Omega_{m}}{D}\right)\right] \\
& \times\left[\frac{3 \varepsilon^{4}}{2 N_{s}}+\frac{\left(7 N_{s}-6\right) \varepsilon^{6}}{3}-f_{1} \varepsilon^{8}+2 f_{1} \varepsilon^{9} F(\varepsilon)\right] \\
& -\frac{\xi_{1} \pi^{1 / 2}}{2\left(t-t_{0}\right)^{7} D^{15 / 2}\left(1+N_{s}\right)^{3 / 2}} \\
& \times\left[\frac{105 \varepsilon^{6}}{32}+\frac{5}{16}\left(11-24 N^{2}{ }_{s}+8 N_{s}\right) \varepsilon^{8}+f_{2} \varepsilon^{10}\right. \\
& \left.+f_{3} \varepsilon^{12}-2 f_{3} \varepsilon^{14} \exp \left(-2 \varepsilon^{2}\right)\left[E_{i}\left(2 \varepsilon^{2}\right)-0.5772\right]+\left(\frac{2 \varepsilon_{m k i} \Omega_{m}}{D}\right)\right] \\
& -\frac{2 \xi_{1} \pi^{1 / 2} N_{s}}{2\left(t-t_{1}\right)^{7} D^{15 / 2} 2\left(1+N_{s}\right)^{3 / 2}} \\
& \times \exp \left\{-\left[\left(1+2 N_{s}\right) \varepsilon^{2}+2+\left(\frac{2 \varepsilon_{m l i} \Omega_{m}}{D}\right)\right]\right. \\
& \times\left\{\frac { 1 0 5 } { 8 N _ { s } } \left[-\frac{\varepsilon^{8}}{3}-\frac{2 \varepsilon^{10}}{3}-\frac{23 \varepsilon^{12}}{30}+\frac{498 \varepsilon^{14}}{630}\right.\right. \\
& \times \exp \left[\left(-2 \varepsilon^{2}\right)+\left(\frac{2 \varepsilon_{m k i} \Omega_{m}}{D}\right)\right]\left[E_{i}\left(2 \varepsilon^{2}\right)-0.5772\right] \\
& +3.1808 \times 10^{-2} \varepsilon^{14}-2.7178 \times 10^{-2} \varepsilon^{16} \\
& +1.3968 \times 10^{-2} \varepsilon^{18}-4.6204 \times 10^{-3} \varepsilon^{20} \\
& +1.1584 \times 10^{-3} \varepsilon^{22}-2.3139 \times 10^{-4} \varepsilon^{24} \\
& +3.8589 \times 10^{-5} \varepsilon^{26}-5.5108 \times 10^{-4} \varepsilon^{28} \\
& +1.1584 \times 10^{-3} \varepsilon^{22}-2.3139 \times 10^{-4} \varepsilon^{24}
\end{aligned}
$$




$$
\begin{aligned}
& \left.+6.8898 \times 10^{-7} \varepsilon^{30}-\ldots\right] \\
& -\frac{15\left(N^{2}{ }_{s}-2 N_{s}-1\right)}{4 N_{s}}\left[-\frac{\varepsilon^{10}}{2}-\frac{5 \varepsilon^{12}}{6}+\frac{14 \varepsilon^{14}}{15}\right. \\
& \times \exp \left[\left(-2 \varepsilon^{2}\right)+\left(\frac{2 \varepsilon_{m k i} \Omega_{m}}{D}\right)\right]\left[E_{i}\left(2 \varepsilon^{2}\right)-0.5772\right] \\
& +2.0261 \times 10^{-2} \varepsilon^{14}+3.5487 \times 10^{-3} \varepsilon^{16} \\
& +5.4047 \times 10^{-4} \varepsilon^{18}+7.2369 \times 10^{-5} \varepsilon^{20} \\
& +8.6172 \times 10^{-6} \varepsilon^{22}+9.22057 \times 10^{-7} \varepsilon^{24} \\
& +8.9477 \times 10^{-8} \varepsilon^{26}+7.9374 \times 10^{-9} \varepsilon^{28} \\
& \left.+6.4814 \times 10^{-10} \varepsilon^{30}+\ldots\right] \\
& +\left(11 N_{s}^{2}-20 N_{s}-10\right) N_{s}\left[-\varepsilon^{12}+\frac{4}{3} \varepsilon^{14}\right. \\
& \times \exp \left[\left(-2 \varepsilon^{2}\right)+\left(\frac{2 \varepsilon_{m k i} \Omega_{m}}{D}\right)\right]\left[E_{i}\left(2 \varepsilon^{2}\right)-0.5772\right] \\
& +8.2505 \times 10^{-2} \varepsilon^{14}+1.7495 \times 10^{-2} \varepsilon^{16} \\
& +3.1572 \times 10^{-3} \varepsilon^{18}+4.90805 \times 10^{-4} \varepsilon^{20} \\
& +6.66926 \times 10^{-5} \varepsilon^{22}+8.0290 \times 10^{-6} \varepsilon^{24} \\
& +8.6642 \times 10^{-7} \varepsilon^{26}+8.4643 \times 10^{-8} \varepsilon^{28} \\
& \left.+7.5495 \times 10^{-9} \varepsilon^{30}+\ldots\right] \\
& -\left(1+2 N_{s}\right) N^{3}{ }_{s}\left[\varepsilon^{14} \exp \left[\left(-\varepsilon^{2}\right)+\left(\frac{2 \varepsilon_{m k i} \Omega_{m}}{D}\right)\right] \times\left[E_{i}\left(2 \varepsilon^{2}\right)-0.5772\right]\right. \\
& +2.2654 \times 10^{-1} \varepsilon^{14}+1.0679 \times 10^{-1} \varepsilon^{16} \\
& -3.3996 \times 10^{-3} \varepsilon^{18}+9.0694 \times 10^{-3} \varepsilon^{20} \\
& -1.1099 \times 10^{-3} \varepsilon^{22}+3.7350 \times 10^{-4} \varepsilon^{24} \\
& -4.444 \times 10^{-5} \varepsilon^{26}+8.2388 \times 10^{-6} \varepsilon^{28} \\
& \left.\left.\left.\left.-8.47486 \times 10^{-7} \varepsilon^{30}+\ldots\right]\right\}\right\}\right\},
\end{aligned}
$$

where

$$
\begin{gathered}
\varepsilon^{2}=D \frac{\left(t-t_{1}\right) k^{2}}{\left(1+N_{s}\right)}, f_{1}=\frac{4}{3}\left(3 N^{2}{ }_{s}-2 N_{s}+3\right), \\
f_{2}=\left(\frac{15-40 N^{2}{ }_{s}\left(2 N_{s}+1\right)+4 N^{2}{ }_{s}\left(11 N^{2}{ }_{s}-20 N_{s}\right)}{16\left(1+N_{s}\right)^{2} D}\right), \\
f_{3}=\left(\frac{15+20\left(4 N^{2}{ }_{s}-2 N_{s}\right)+4 N^{2}\left(9 N^{2}{ }_{s}-20 N_{s}\right)-16 N^{2}}{8\left(1+N_{s}\right)^{3}}\right) .
\end{gathered}
$$

In a turbulent phenomenon, such as turbulent kinetic energy, we associate the so-called concentration energy with the fluctuating concentration, defined by the relation

$$
\frac{1}{2}\left\langle X X^{\prime}\right\rangle=\int_{-\infty}^{\infty} G(k, t) d k
$$

The substitution of equation (28) and subsequent integration with respect to $\mathrm{k}$ leads to the result

$$
\begin{gathered}
\frac{1}{2}\left\langle X^{2}\right\rangle=\exp \left[-2 R\left(t-t_{0}\right)\right] \\
\times\left[\begin{array}{l}
A\left(t-t_{0}\right)^{-\frac{3}{2}}+B\left(t-t_{0}\right)^{-5} \exp \left[-R_{2}\left(t-t_{0}\right)\right] \\
-\left\{\begin{array}{l}
C\left(t-t_{0}\right)^{\frac{-15}{2}}-D_{1}\left(t-t_{0}\right)^{-\frac{15}{2}}\left(t-t_{1}\right)^{\frac{1}{2}}-E\left(t-t_{0}\right)^{-15} \\
\left.-F\left(t-t_{0}\right)^{-\frac{29}{2}}-G\left(t-t_{0}\right)^{-15}\left(t-t_{1}\right)^{\frac{1}{2}}\right\} \exp \left[-R_{3}\left(t-t_{0}\right)\right]
\end{array}\right]
\end{array}\right.
\end{gathered}
$$

This is the decay law of first order reactant in homogeneous fluid turbulence in a rotating system, where

$$
A=\frac{N_{0}}{D^{3 / 2} 2^{7 / 2} \pi^{1 / 2}}, B=\frac{\xi_{0} R_{1}}{D^{6}}, C=c d,
$$

$$
\begin{gathered}
D_{1}=c e, E=c f^{\prime}\left(g h+g j_{1}-k_{1} l_{1}-k_{1} n+p q+p s-t^{\prime} v w\right), \\
F=c f^{\prime}\left(g i_{1}-k_{1} m-h t^{\prime}\right), G=c f^{\prime} p r_{1}, \\
c=\frac{15 \pi \xi_{1}}{D^{8} 2^{\frac{15}{2}} N_{s}^{\frac{7}{2}}\left(1+N_{s}\right)},
\end{gathered}
$$$$
d=\frac{105}{32}+\frac{35\left(11-24 N^{2}+8 N_{s}\right)}{64 N_{s}}+\frac{63 f_{2}}{8 N_{s}^{2}}+\frac{693 f_{3}}{64 N_{s}^{3}}-\frac{1808 f_{3}}{256}+\frac{41.5910 f_{3} N^{\frac{7}{2}} s}{\left(1+N_{s}\right)^{\frac{1}{2}}},
$$$$
e=2 f_{3} \frac{D^{\frac{1}{2}} I\left(-2 N_{s} \varepsilon^{2}, \varepsilon\right)^{2}}{\left(1+N_{s}\right)^{\frac{1}{2}} \pi^{\frac{1}{2}}}, f^{\prime}=\frac{37.12311 \xi_{1} N_{s} \pi^{\frac{1}{2}}}{D^{8} \varepsilon^{\frac{9}{2}}\left(1+N_{s}\right)}, g=\frac{105}{2 N_{s}},
$$$$
h=-\frac{1}{3}-\frac{6}{\varepsilon_{1}}-\frac{75.90}{\varepsilon^{2}{ }_{1}}-\frac{2.52052 \times 10^{-3} \varepsilon_{1}^{\frac{9}{2}}}{\left(1+N_{s}\right)^{\frac{15}{2}}},
$$

$$
\left.i_{1}=1.118 \varepsilon_{1}^{\frac{9}{2}} \frac{\left(1+N_{s}\right)^{\frac{1}{2}}}{D} \times I\left[\left(1+2 N_{s}\right) \mathcal{E}, \varepsilon^{2}\right]\right),
$$

$$
\begin{gathered}
j_{1}=\frac{163}{\varepsilon_{1}^{3}}\left(3.1808 \times 10^{-2}-4.0767 \times 10^{-1} \varepsilon_{1}^{-1}+3.561804 \varepsilon_{1}^{-2}\right. \\
-2.2386 \times 10 \varepsilon_{1}^{-3}+1.1786 \times 10^{2} \varepsilon_{1}^{-4}-5.4148 \times 10^{2} \varepsilon_{1}^{-5} \\
\left.+2.02576 \times 10^{3} \varepsilon_{1}^{-6}-8.7048 \times \varepsilon_{1}^{-7}+3.1561 \times 10^{4} \varepsilon_{1}^{-8}-\ldots\right), \\
k_{1}=\frac{135\left(6 N_{s}^{2}-2 N_{s}-1\right)}{N_{s} \varepsilon_{1}}, \\
l_{1}=-\frac{1}{2}-\frac{55}{6 \varepsilon_{1}}-\frac{2.3510 \times 10^{-3} \times 163 \varepsilon_{1}^{\frac{11}{2}}}{2^{15}\left(1+N_{s}\right)^{\frac{15}{2}}}
\end{gathered}
$$




$$
\begin{aligned}
& \left.m=7.4469 \varepsilon_{1}^{\frac{11}{2}} \times \frac{\left(1+N_{s}\right)^{-\frac{1}{2}}}{D} I\left[\left(1+2 N_{s}\right) \varepsilon^{2}, \varepsilon^{2}\right]\right), \\
& n=\frac{163}{\varepsilon_{1}^{2}}\left(2.0261+5.3230 \times 10^{-2} \varepsilon_{1}^{-1}\right. \\
& +1.3782 \times 10^{-1} \varepsilon_{1}^{2}+3.5063 \times 10^{-1} \varepsilon_{1}^{-3} \\
& +8.7676 \times 10^{-1} \varepsilon_{1}^{-4}+2.57710^{-1} \varepsilon_{1}^{-5} \\
& \left.+5.2347 \varepsilon_{1}^{-6}+1.2538 \varepsilon_{1}^{-7}+2.9690 \times 10 \varepsilon_{1}^{-8}+\ldots\right), \\
& p=396\left(11 N_{s}^{2}-20 N_{s}-10\right) N_{s} \varepsilon_{1}^{-2}, \\
& q=-1-3.0532 \times 10^{-4} \varepsilon_{1}^{\frac{13}{2}}\left(1+N_{s}\right)^{\frac{-15}{2}}, \\
& r_{1}=1.0638 \varepsilon_{1}^{\frac{13}{2}}\left(t-t_{1}\right)^{\frac{1}{2}} \frac{\left(1+N_{s}\right)^{-\frac{1}{2}}}{D} \times I\left[\left(1+2 N_{s}\right) \varepsilon^{2}, \varepsilon^{2}\right], \\
& s=13 \varepsilon_{1}^{-1}\left(8.2504+2.6243 \times 10^{-1} \varepsilon^{-1}+8.0509 \times 10^{-1} \varepsilon^{-2}\right. \\
& +2.3780 \times \varepsilon_{1}^{-3}+6.7856 \varepsilon_{1}^{-4}+1.8789 \times 10 \varepsilon_{1}^{-5} \\
& \left.+5.0688 \times 10^{1} \varepsilon_{1}^{-6}+1.3370 \times 10^{2} \varepsilon_{1}^{-7}+3.4583 \times 10^{2} \varepsilon_{1}^{-8}+\ldots\right), \\
& t^{\prime}=-1.4219 N_{s}^{2} \varepsilon_{1}^{\frac{11}{2}}\left(1+N_{s}\right)^{\frac{1}{2}}, \\
& u=\left\{\left(-3.1886 \times 10^{-3}+1.4443 \times 10^{2}\left(t-t_{0}\right)^{\frac{1}{2}}\right.\right. \\
& \left.\left.\times D^{\frac{1}{2}}\left(1+N_{s}\right)^{-\frac{1}{2}} I\left[\left(1+2 N_{s}\right) \varepsilon^{2}, \varepsilon^{2}\right]\right)\right\}, \\
& v=1.8102 \times 10^{2}\left(1+2 N_{S}\right)^{\frac{1}{2}} \varepsilon_{1}^{-\frac{15}{2},} \\
& w=\left(2.2653 \times 10^{-1}+1.60195 \varepsilon_{1}^{-1}\right. \\
& -8.6664 \times 10^{-1} \varepsilon^{-2}+4.3941 \times 10 \varepsilon_{1}^{-3} \\
& -1.1293 \times 10^{2} \varepsilon_{1}^{-4}+8.7405 \times 10^{2} \varepsilon_{1}^{-5} \\
& -2.6001 \times 10^{3} \varepsilon_{1}^{-6}+1.3013 \times 10^{4} \varepsilon_{1}^{-7} \\
& \left.-3.8822 \times 10^{4} \varepsilon_{1}^{-8}+\ldots\right) \\
& R_{1}=\frac{\pi}{2\left(1+N_{S}\right)\left(1+2 N_{s}\right)^{\frac{5}{2}}}\left[\frac{9}{16}+\frac{5 N_{s}\left(7 N_{s}-6\right)}{16\left(1+2 N_{s}\right)}\right. \\
& \left.-\frac{105 f_{1} N_{s}}{32\left(1+2 N_{s}\right)}+\frac{f_{1} N_{s}\left(1+2 N_{s}\right)^{\frac{5}{2}}}{2^{\frac{21}{2}}\left(1+N_{s}\right)^{\frac{11}{2}}} \times \sum_{n=0}^{\infty} \frac{1.3 .5 \ldots(2 n+9)}{n !\left(2 N_{s}+1\right) 2^{2 n}\left(1+N_{s}\right)^{n}}\right] \\
& \varepsilon_{1}=2\left(1+2 N_{s}\right), R_{2}=R_{3}=\left(\frac{2 \varepsilon_{m k i} \Omega_{m}}{D}\right),
\end{aligned}
$$

and

$$
I\left[-\alpha \varepsilon^{2}, \beta \varepsilon^{2}\right]=\int_{0}^{\infty} \exp \left(-\alpha \varepsilon^{2}\right)\left[\varepsilon^{14} \exp \left(\beta \varepsilon^{2}\right) E_{i}\left(\beta \varepsilon^{2}\right)\right] d k
$$

The first term of equation (30) corresponds to the concentration energy for two-point concentration; the second term represents first order reactant in homogeneous fluid turbulence in a rotating system for three-point correlation. The expression $\exp \left[-R_{2}\left(t-t_{0}\right)\right]$ represents the fluid turbulence in a rotating system for three-point correlation, $\exp \left[-R_{3}\left(t-t_{0}\right)\right]$ and the remainders are due to four-point correlation.

\section{Results and Discussion}

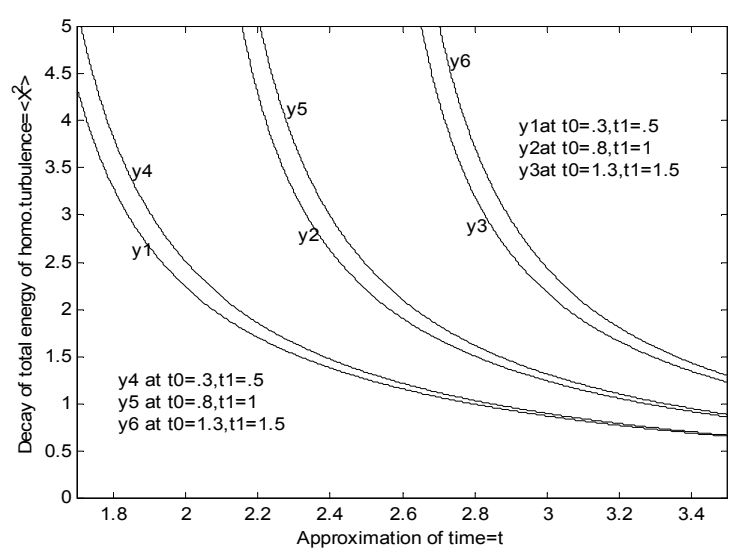

Fig. 1. Comparison between equation (30) and equation (31) if $R=0.025$.

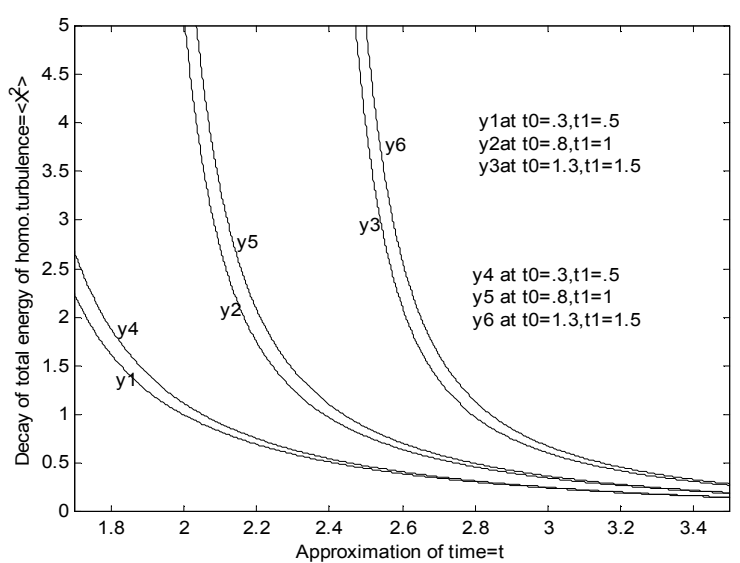

Fig. 2. Comparison between equation (30) and equation (31) if $R=0.5$.

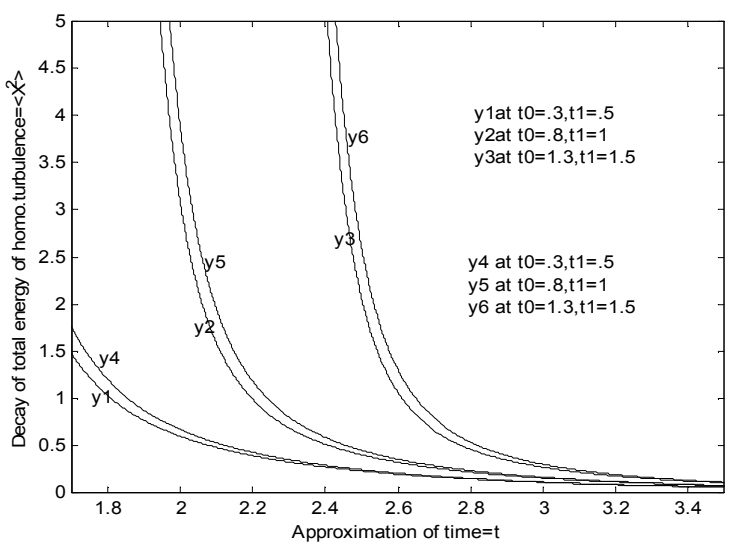

Fig. 3. Comparison between equation (30) and equation (31) if $R=0.8$. 


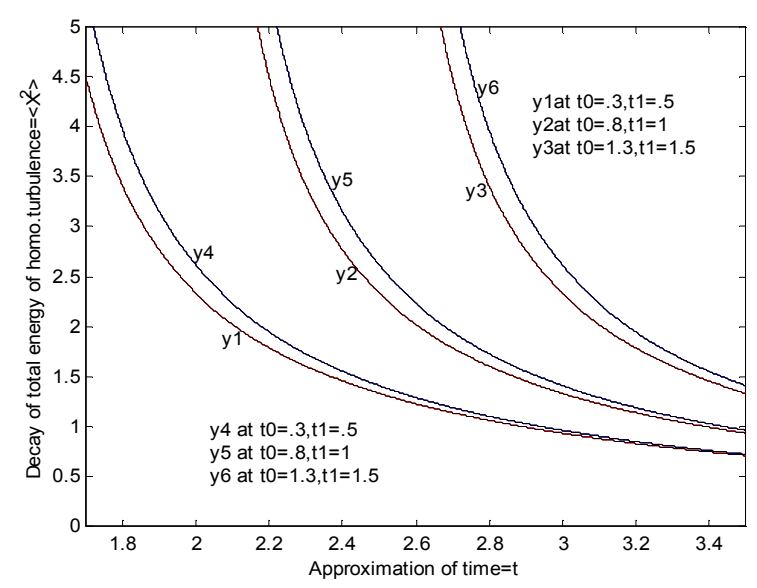

Fig. 4. Comparison between equation (30) and equation (31) if $R=0$.

$$
\begin{aligned}
& \frac{1}{2}\left\langle X^{2}\right\rangle=\exp \left[-2 R\left(t-t_{0}\right)\right] \times\left[A\left(t-t_{0}\right)^{-\frac{3}{2}}+B\left(t-t_{0}\right)^{-5}\right. \\
& \left.-\left\{C\left(t-t_{0}\right)^{\frac{-15}{2}}-D_{1}\left(t-t_{0}\right)^{-\frac{15}{2}}\left(t-t_{1}\right)^{\frac{1}{2}}-E\left(t-t_{0}\right)^{-15}-F\left(t-t_{0}\right)^{-\frac{29}{2}}-G\left(t-t_{0}\right)^{-15}\left(t-t_{1}\right)^{\frac{1}{2}}\right\}\right]
\end{aligned}
$$

this is obtained by Kumar and Patel (1974). Here $A, B, C, D_{1}$, $E, F, G$ are an arbitrary constants. With $\mathrm{R}=0$ and the contaminant replaced by the temperature, the results show complete argument with the result obtained by Loeffler and Deissler (1961) for the decay of temperature fluctuation in homogeneous turbulence before the final period up to three-point correlations. For large times, the last terms become negligible and give the $-3 / 2$ power decay law for the final period. In figures, Eq. (30) represented by the curves y1, $\mathrm{y} 2, \mathrm{y} 3$ and (31) by $\mathrm{y} 4, \mathrm{y} 5, \mathrm{y} 6$. For $R_{2}=0.25$, the comparison between the Eq. (30) and (31) are shown in Fig.1, Fig.2, Fig.3 and Fig.4 corresponding to the values $R=.025, .5, .8$ and 0 respectively, which indicated in the figures clearly.

\section{Conclusion}

From the figures and discussions, this study shows that the chemical reaction $(\mathrm{R}=0)$ of the first exponential factor of equation (30) and (31) causes the concentration fluctuation to decay more rapidly than they would for $(R \neq 0)$ non pure mixing and due to the effect of rotation of homogeneous fluid turbulence in the flow field of the first order chemical reaction for four-point correlation, the turbulent energy decays more slowly than the energy decay for first order reactant in homogeneous turbulence.

\section{References}

[1] Aziz, M.A, M.A.K. Azad and M.S. Alam Sarker, "First order reactant in MHD turbulence before the final period of decay for the case of multi-point and multi-time in a rotating system in presence of dust particle," Res. J. Math. Stat. vol. 2, pp. $56-68,2010$.
In equation (30) we obtained the decay law of first order reactant in homogeneous fluid turbulence for four-point correlation in a rotating system after neglecting quintuple correlation terms. The equation contains the terms $\left(t-t_{0}\right)^{-3 / 2},\left(t-t_{0}\right)^{-5},\left(t-t_{0}\right)^{-15 / 2}$ Thus, the terms associated with the higher-order correlation die out faster than those associated with the lower-order ones; therefore, the assumption that the higher-order correlations can be neglected in comparison with lower-order correlations seems to be valid in our case. If the system is non-rotating (i.e., $\Omega_{m}=0$ ) the equation (30) becomes
[2] Batchelor, G.K., "The Theory of Homogeneous Turbu lence." Cambridge University press, Cambridge, 1953. pp: 79.

[3] Bkar Pk, M.A., M.A.K. Azad and M.S.Alam Sarker, "Decay of energy of MHD turbulence for four-point correlation." International Journal of Engineering \& Technology.1 (9):pp1-13. 2012.

[4] Bkar Pk. M.A., M.A.K.Azad and M.S.Alam.Sarker, "First-order reactant in homogeneou turbulence prior to the ultimate phase of decay for four-point correlation in presence of dust particle,"Res.J.Appl.Sci.Eng. Technol.,5(2):585-595. 2013(a).

[5] Bkar Pk, M.A., M.S.Alam Sarker and M.A.K. Azad, "Decay of MHD turbulence before the final period for four-point correlation in a rotating system.” Res. J. Appl. Sci. Eng. Technol., 6(15), 2789-2798. 2013(b).

[6] Chandrasekhar, S., "The invariant theory of isotropic turbulence in magneto-hydrodynamics," Proc. Roy.Soc., London, and A204:435-449. 1951.

[7] Corrsin, S., "On the spectrum of isotropic temperature fluctuations in isotropic turbulence.J.Apll.Phys,".J.Apll.Phys 22:469-473.1953.

[8] Deissler, R.G., "On the decay of homogeneous turbulence before the final period.” Phys.Fluid, 1:111-121. 1958.

[9] Deissler, R.G., "A theory of decaying homogeneous turbu lence.” Phys. Fluid, 3:176-187. 1960.

[10] Islam, M.A. and M.S.A Sarker, "First order reactant in MHD turbulence before the final period of decay for the case of multi-point and multi-time." Indian J. pure appl. Math., 32(8):1173-1184. 2001.

[11] Kumar, P. and S.R.Patel, "First-order reactant in homogeneous turbulence before the final period of decay." Phys .Fluids, 17: 1362-1368. 1974.

[12] Kumar, P. and S.R.Patel, "On first-order reactant in homogeneous turbulence.’Int. J. Eng. Sci., 13:305-315. 1975. 
[13] Loeffler, A.L and R.G. Deissler, "Decay of temperature fluctuations in homogeneous turbulence before the final period.” Int. J. Heat Mass Trans., 1: 312-324. 1961.

[14] Mondal, M.N.I., "Energy decay law of dusty fluid turbulent flow in a rotating system.” J. Eng. Applied Sci., 1:71. 2006.

[15] Sarker, M. S. A. and N. Kishore, "Decay of MHD turbulence before the final period.” Int. J. Engng Sci., 29(11):1479-1485. 1991.
[16] Sarker, M. S. A. and M.A. Islam, "Decay of dusty fluid MHD turbulence before the final period in a rotating system." J. Math. \& Math. Sci, 16:35. 2001.

[17] Sultan, S., "Energy decay law of dusty fluid turbulent flow in a rotating system." J.Eng. Applied Sci., 3:166. 2008. 